\title{
The Contribution of Health Education to the Falling Incidence within Hospital Premises in China
}

\author{
Qiuyao He ${ }^{1 *}$, Jing Zhang'2 Xin Chen ${ }^{3}$ \\ 1West China Hospital, Sichuan University, Chengdu 610041, Sichuan Province, China \\ ${ }^{2}$ Jiangsu College of Nursing, Huaian 223300, Jiangsu Province, China \\ ${ }^{3}$ University Hospitals Coventry and Warwickshire NHS Trust, West Midlands, CV1 5QP, UK \\ *Corresponding author: Qiuyao He, heq13@uni.coventry.ac.uk
}

\begin{abstract}
Falling is the second leading cause of accidental injury to death worldwide. It is estimated that more than $80 \%$ of the people die each year in low and middle-income countries. Falling is also the leading cause of death among the elderly in China. Among them, the falling rate in 2016 increased by 5\% compared with 2006 . Although not fatal, those who fell and have a disability, which may face significant economic risks associated with subsequent long-term care needs, especially for the elderly ${ }^{[1]}$. It may be essential to use evidence-based guidelines for proving health education to address the issue of preventing hospitalization for the elderly. This article will combine the author's practical experience to examine the contribution of health education to prevent falls and explore its application and development in China. Lastly, it will explain barriers to health education.
\end{abstract}

Keywords: Health education; Incidence; Falls; China

Publication date: July 2021; Online publication: July 31, 2021

\section{Introduction}

International anti-fall interventions have demonstrated their contribution to preventing falls, such as sedatives, fall risk assessment tools, and fall markers ${ }^{[2-3]}$. However, health education is regarded as the primary intervention to prevent falls in the elderly combined with Chinese culture, which is also in line with the concept of person-centered quality care projects in China's recent years ${ }^{[4]}$. Health education is not a new concept. It is used as a tool for managing care programs. It may include general preventive education or health promotion and education for disease status ${ }^{[5]}$, especially in nursing practice, to prevent falls ${ }^{[6]}$. At present, there is no relevant health education guideline for hospitalized elderly patients in China. Still, many hospitals refer to advanced instructions from developed countries but combined with the cultural context in clinical practice ${ }^{[7]}$. Moreover, the China Ministry of Health's, "Prevention Technical Guidelines on Fall of Old People" also clearly pointed out that health education as an intervention method for international certification can effectively avoid the fall of the elderly ${ }^{[8]}$. Additionally, May 9 th is designated as the National Health Priority Plan Day in China ${ }^{[8]}$. the program aims to explain to older people about health knowledge in preventing falls ${ }^{[8]}$. The implementation of this policy might be a positive driver of health education intervention ${ }^{[8]}$. The process of clinical care decision-making requires nurses to develop health education content related to the care plan based on the patient's disease needs and reasonable subjective wishes ${ }^{[9]}$. The author uses Chinese health policy, international guidelines, and practical experience to explore the contribution of health education to preventing falls in elderly inpatients ${ }^{[10]}$. Since, the evidence-based practice requires nurses to carefully and intelligently apply care practices during the 
planning, thus the execution of care activities are based on the best evidence, practice guidelines, standards, evidence databases, and clinical experience provided by the investigator.

\section{Nurses to use anti-fall education tools for inpatients in China: videos, leaflets}

The positive impact of fall prevention due to the continuous development and application of fall prevention education tools, may prevent accidental falls in hospitals by improving the fall prevention behavior of older inpatients and increasing their awareness ${ }^{[11]}$. However, Ma, Chan and Chen, doubt the effectiveness of using new technologies in elderly care practices ${ }^{[12]}$, as many Chinese elderly patients could not understand the use of new technologies because of lower levels of education ${ }^{[13]}$. Even though the existing anti-fall education videos are long and the quality of the content is questionable ${ }^{[14]}$, a quasi-experimental study showed a significant improvement in the awareness of falling risk among older patients in the video education intervention group $(\mathrm{P}=0.021)$. Furthermore, the content and format of the video have satisfactory content validity. Also, another quasi-experiment proved that the leaflet education plus video education improved the fall prevention behavior of elderly inpatients $(\mathrm{P}<0.001)$ and effectively improved the positive attitude of the hospitalized elderly against falls $(\mathrm{P}<0.001)$. Moreover, this quasi-experimental study also correlated the satisfaction of hospitalized elderly with video and leaflet education higher than that of videoonly education or leaflet-only education. Since video plus leaflet education might be most effective in improving preventive behavior. Kang and Song suggested that it could be included in the fall prevention education guidance for elderly inpatients ${ }^{[15]}$.

\section{Chinese nursing practice uses teach-back and education program as health education tools}

Health education may avoid unintentional falls by increasing the awareness of inpatients' fall prevention ${ }^{[16]}$. Although when questioned the contribution of teach-back in preventing falls in hospitalized patients. As nurses once provided procedures and rationale, they would consistently use and document education to prevent falls, so they did not use evidence-based methods to improve communication with patients. However, the simplicity and the potential care effects of teach-back still deserve further evidence-based research ${ }^{[17]}$. Adults who are hospitalized have a high risk of falling during acute care, so making the prevention education program has been a critical element of nursing care ${ }^{[18]}$. A quasi-experimental study showed that multimedia procedures by nurses showed an increased level of awareness of falling risk among hospitalized adults ${ }^{[19]}$. Shanghai Sunshine Rehabilitation Center, which was established in 2007, provides health education knowledge for the prevention of falls for hospitalized elderly patients ${ }^{[20]}$. The results show that such a health education team, not only makes elderly inpatients aware of the importance of risk factors and determinants associated with falls, but also more likely to take action to correct adverse health and safety behaviors. Moreover, this supervision process involves not only the participation of nurses but also doctors and rehabilitation therapists. The reliability of the fall prevention knowledge provided to patients through different health professional perspectives could be higher than that of nurses as the sole performer ${ }^{[21]}$. It is somewhat similar to the "Excercise Education Program" from Hong Kong. The only difference is that this program provides exercise knowledge for the elderly to prevent falls ${ }^{[22]}$. Additionally, a randomized controlled trial study also showed the effectiveness of the program in preventing falls in older adults. It illustrated that the risk of falling for participants was from moderate to minimal $(\mathrm{P}<0.001)$ and increased scores of balance confidence, which reduced the risk of falls. Another multicenter randomized controlled trial from Taiwan also showed the positive impact of a multifactorial fall prevention program on preventing falls in elderly patients ${ }^{[23]}$. The results of the study showed that the incidence of falls in the elderly group who received the intervention was reduced by $2.4 \%$ through increasing the patient's anti-fall awareness. Therefore, interventions through these health education programs can avoid unintentional falls by increasing the patient's knowledge of the risk factors for falls and increasing the patient's anti-fall 
awareness.

\section{Nurse-led health education programs for patients and their caregivers or family members}

In many cultures, falling is considered a normal phenomenon of aging, which is an inevitable result. The results of a study showed that $88 \%$ of hospitalized older adults believe that they do not have the risk of falling ${ }^{[24]}$. Therefore, this requires nurses to remind patients' caregivers to fill in the gap when delivering knowledge about health education to prevent falls. Also, many families with patients who have cognitive impairment are reluctant to send them to nursing homes but hospitals because of filial piety culture under the influence of Chinese Confucian culture, such as dementia. Therefore, nurses can provide knowledge about the prevention of falls for caregivers or family members with dementia ${ }^{[25]}$. Besides, the nurse reminds the patient's caregivers or family members that the patient with the risk of falling should be an essential regulatory goal in the health education process ${ }^{[26]}$. Currently, quality nursing service projects also clarifies the importance of health education to prevent accidental falls in elderly patients in China. A quasiexperimental study from Hong Kong showed that providing prevention of falls to caregivers or family members with a high risk of falls can effectively reduce the incidence of falls by nearly $10 \%$, such as chronic illnesses without walking ability ${ }^{[27]}$. Another study showed that the nurse-led patient safety quality improvement program reduced the fall rate by $29.4 \%$. The program is designed to prevent falls during hospitalization by nursing staff, providing videos of fall prevention knowledge to patients and their families. Therefore, nurses are not only responsible for improving the patient's self-awareness of falling prevention, but equally important, to promote the participation of patient caregivers or their families to ensure that they prevent the falling by providing them with knowledge about falls ${ }^{[28]}$.

\section{Content of health education for preventing falls with Chinese cultural characteristics: Tai Chi}

The nurse may inform the inpatient that Tai Chi can prevent unintentional falls through muscle exercise and improve balance coordination ${ }^{[29]}$. However, a study has questioned the contribution of Tai Chi to the prevention of falls ${ }^{[30]}$. Nevertheless, the education team still guides patients to use Tai Chi to prevent falls, as this exercises elderly patients' muscles and reduces the risk of falls. Nurses from Chinese medicine hospitals not only provide information to patients that Tai Chi could reduce the risk of falling by balancing physical coordination, but also teaches patients how to properly do Tai Chi in a monthly departmental health education program. Moreover, a systematic review also demonstrated a significant reduction in the risk of falls in the Tai chi group (odds ratio $=0.70 ; 95 \%$ confidence interval 0.59 to 0.84$)^{[31]}$. Moreover, a study also suggested that further research is needed for Tai Chi exercise to develop the best Tai Chi training program (training intensity, duration and frequency) for the elderly ${ }^{[31]}$. Therefore, the author believes that it is necessary for nurses to provide the elderly with the contribution of Tai chi to prevent falls through muscle exercise and balance coordination ${ }^{[30]}$.

\section{Nurse's face barriers in implementing health education: culture, nurse personal knowledge}

Many elderly inpatients are very stubborn under the influence of the "Chinese Confucian" culture. As they only recognize their own experience, and may not trust the advice from young nurses, thus the author combined clinical experience suggesting that nursing managers assume the role of health education may effectively deepen the awareness of falls in older patients in China ${ }^{[32]}$. Since nursing managers have professional fall prevention knowledge, skills and excellent communication skills, thus according to the author's practical experience, the nurse's knowledge system may influence the effectiveness of nursing activities. Therefore, nurse educators and managers should ensure that nurses are thoroughly familiar with the assessment of falls. They should enhance the knowledge structure of nurses through continuing education ${ }^{[33]}$. Also, health education is organizational culture, and the active leadership style of caregivers 
may contribute to the establishment of this culture. A culture that is conducive to patient safety is promoted based onward care leaders, and nurses are encouraged to build more evidence bases for fall prevention. These two cultures are also in line with quality care programs in China.

\section{Conclusion}

In conclusion, health education has been widely promoted as an effective anti-fall nursing intervention in China. The author examined the application and development of health education to prevent falls in nursing practices in China. It mainly evaluated the tools, methods, target groups and content effectiveness of health education. Nevertheless, it has limitations which not only require further, but also extensive research. Thus, this may require more engagement from leaders and national policy. In general, nurses need to ensure that critical thinking skills and evidence are integrated into care activity decisions to improve the quality of care.

\section{Disclosure statement}

The author declares no conflict of interest.

\section{References}

[1] Boyé N, Van L, Esther MM, et al., 2013, The Impact of Falls in the Elderly. Trauma, 15(1): 29-35.

[2] Smith ML, Towne SD, Motlagh AS, et al., 2017, Programs and Place: Risk and Asset Mapping for Fall Prevention. Frontiers in Public Health, 5:28.

[3] Nelson-Wong E, Appell R, McKay M, et al., 2012. Increased Fall Risk is Associated with Elevated Co-Contraction about the Ankle During Static Balance Challenges in Older Adults. European Journal of Applied Physiology, 112(4):1379-1389.

[4] Zhang L, Dalal K, Yin M, et al., 2012, The KAP Evaluation of Intervention on Fall-Induced Injuries among Elders in a Safe Community in Shanghai, China. PLoS ONE, 7(3):96-105.

[5] Whitehead D, 2008, An International Delphi Study Examining Health Promotion and Health Education in Nursing Practice, Education and Policy. Journal of Clinical Nursing, 17(7): 891-900.

[6] Scott V, Gallagher E, Higginson A, et al., 2011, Evaluation of an Evidence-Based Education Program for Health Professionals: The Canadian Falls Prevention Curriculum $\subset$ (CFPC). Journal of Safety Research, 42(6):501-507.

[7] National Institute for Health and Care Excellence, 2013, Falls in Older People: Assessing Risk and Prevention. https://www.nice.org.uk/guidance/cg161/resources/ pdf-35109686728645

[8] General Office of the State Council of the People's Republic of China, 2016, Healthy China 2030 Planning Outline. http://www.gov.cn/zhengce/2016-10/25/content_5124174.htm>

[9] Parrish DE, 2018, Evidence-Based Practice: A Common Definition Matters. Journal of Social Work Education, 54(3):407-411.

[10] Mullen EJ, 2016, Reconsidering the Idea of Evidence in Evidence-Based Policy and Practice. European Journal of Social Work, 19(3-4):310-335.

[11] Dilley L, Gray S, Zecevic A, et al., 2014, An Educational Video to Promote Multi-Factorial Approaches for Fall and Injury Prevention in Long-Term Care Facilities. BMC Medical Education, 14(1):102.

[12] Ma Q, Chan AHS, Chen K. 2016, Personal and Other Factors Affecting Acceptance of Smartphone Technology by Older Chinese Adults. Applied Ergonomics, 54:62-71. 
[13] Lin S, Tseng WT, Hsu M, et al., 2017, A Psychometric Evaluation of the Chinese Version of the Nursing Home Survey on Patient Safety Culture. Journal of Clinical Nursing, 26(23-24): 46644674.

[14] Wan YA, Neni H, Siew TL, et al., 2018, Evaluation of a Fall Prevention Educational Video on Fall Risk Awareness, Knowledge and Help Seeking Behaviour Among Surgical Patients, Singapore Nursing Journal, 45(1):27-33.

[15] Kang YO, Song R, 2018, Effects of Fall Prevention Education Program on Attitudes, Prevention Behaviors, and Satisfaction among Elderly Inpatients. Korean Journal of Adult Nursing, 30(1):4959.

[16] Zhu Y, Ghajar M, Mitre E, 2016, SHARE: Spreading Health Awareness with Resources and Education-Librarians' Role in Patient Education, A Case Study. Journal of Hospital Librarianship, 16(4):319-327.

[17] Klingbeil C, Gibson C, 2018, The Teach Back Project: A System-wide Evidence Based Practice Implementation. Journal of Pediatric Nursing, 42:81-85.

[18] Mackay M, Joyce-Mccoach J, Stephens M, et al., 2016, Review of Transnational Nursing Education Programme Curricula: Process, Findings, and Recommendations. Nurse Education Today, 42:7377.

[19] Cerilo PC, 2016, Effectiveness of Fall Prevention Multimedia Program on Patient Awareness, SelfEfficacy, and Engagement, Effectiveness of Fall Prevention Multimedia Program on Patient Awareness, Self-Efficacy \& Engagement, p.1.

[20] Wang X, Chontawan R, Nantsupawat R, 2012, Transformational Leadership: Effect on the Job Satisfaction of Registered Nurses in a Hospital in China. (Report). Journal of Advanced Nursing, 68(2):444-451.

[21] Zhang H, Shen Q, 2014, The Current Situation of Rehabilitation Medical Service System in China: Problems and Challenges. Business and Management Research, 3(2):42-46.

[22] Anderson J, Browne A, Reimer-Kirkham S, et al., 2010, Uptake of Critical Knowledge in Nursing Practice: Lessons Learned from a Knowledge Translation Study. Canadian Journal of Nursing Research, 42(3):106-122.

[23] Lee H, Chang K, Tsauo J, et al., 2013, Effects of a Multifactorial Fall Prevention Program on Fall Incidence and Physical Function in Community-Dwelling Older Adults With Risk of Falls. Archives of Physical Medicine and Rehabilitation 94(4):606-15.

[24] Sonnad SS, Mascioli S, Cunningham J, et al., 2014, Do Patients Accurately Perceive Their Fall Risk?, Nursing, 44(11):58-62.

[25] Wu B, Darina VP, Jing W, et al., 2019, Dementia Caregiver Interventions in Chinese People: A Systematic Review. Journal of Advanced Nursing 75(3):528-542.

[26] Driscoll J, Stacey G, Harrison-Dening K, et al., 2019, Enhancing the Quality of Clinical Supervision in Nursing Practice. Nursing Standard, 34(5):43-50.

[27] Xia Q, Jiang Y, Niu CJ, et al., 2009, Effectiveness of a Community-Based Multifaceted FallPrevention Intervention in Active and Independent Older Chinese Adults. Injury Prevention, 15(4):248-24851.

[28] You LM, Liu K, He G, et al., 2013, Hospital Nursing, Care Quality, and Patient Satisfaction: CrossSectional Surveys of Nurses and Patients in Hospitals in China and Europe. International Journal of Nursing Studies, 50(2):154-161. 
[29] Luk JK, Chan TY, Chan D, 2015, Falls Prevention in the Elderly: Translating Evidence into Practice. Hong Kong Medical Journal of Xianggang Yi Xue Za Zhi, 21(2):165-171.

[30] Gallant M, Tartaglia M, Hardman S, Burke K, 2019, Using Tai Chi to Reduce Fall Risk Factors Among Older Adults: An Evaluation of a Community-Based Implementation. Journal of Applied Gerontology, 38(7):983-998.

[31] Hu YN, Chung YJ, Yu HK, et al., 2016, Effect of Tai Chi Exercise on Fall Prevention in Older Adults: Systematic Review and Meta-analysis of Randomized Controlled Trials. International Journal of Gerontology 10(3):131-36.

[32] Wang L, Ning P, Peng Y, et al., 2019, Unintentional Falls Mortality in China, 2006-2016. Journal of Global Health, 9(1):010603.

[33] Zhang F, Tian L, Shang X, Li, X., et al., 2018, Exploring Relationships Between First-Line Nurse Manager's Safety Attitudes and Safety Factors in Henan, China. Journal of Nursing Management, 26(3), 14-320. 